\section{KOMPASS}

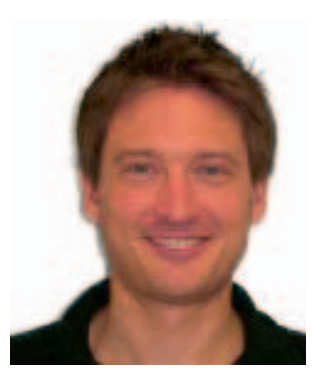

A. Rembert Koczulla

Klinik für Pneumologie,

Philipps-Universität Marburg

Marburg, Deutschland

\title{
Obstruktive Lungenerkrankungen: personalisierte Medizin bei Asthma und COPD
}

Im Januar 2015 hat der damalige US-amerikanische Präsident Barack Obama die breite Öffentlichkeit erstmals darüber in Kenntnis gesetzt, dass er neue innovative Wege in der Medizin beschreiten möchte.

Er prägte mit diesem Vorstoß den Begriff «precision medicine», die eine neue Ära in der Medizin einleiten sollte. Dabei sollen Forscher, Gesundheitsdienstleister und $\mathrm{Pa}$ tienten zusammenarbeiten, um eine individuelle Versorgung des Einzelnen möglich zu machen. Dafür wurden Summen von mehr als 200 Millionen Dollar im US-Haushalt vorgesehen mit dem Ziel, die Gesundheit der US-amerikanischen Bürger und die Behandlung von Krankheiten maßgeblich zu verbessern.

Der Begriff Präzisionsmedizin oder personalisierte Medizin ist auch in Deutschland mittlerweile ein großes Thema und spielt ebenso im internistisch-pneumologischen Bereich wie z.B. bei Asthma und bei chronisch-obstruktiver Lungenerkrankung (chronic obstructive pulmonary disease, (OPD) eine Rolle.

Es wird bereits nach sensitiven und spezifischen Biomarkern gesucht, die letztendlich eine bessere und zielgerichtete Auswahl bestehender Therapieoptionen für sehr gut charakterisierte Patienten ermöglichen.

Bei aller Fokussierung auf neue Marker im Rahmen der Präzisionsmedizin sollte aber nicht außer Acht gelassen werden, dass - wie in einer allgemeinmedizinischen Arbeit gezeigt wurde - beispielsweise die Anamnese knapp 80\% der Diagnosen ermöglicht [1]. Ein großes Problem in der heutigen Medizin ist sicherlich, dass häufig die Zeit fehlt, um eine genaue medizinische Anamnese zu erheben, wenn man bedenkt, dass aktuell statistisch nur noch 6-7 Minuten pro Patientenkontakt zur Verfügung stehen.

So sind ganz einfache Dinge, wie das Alter bei Erstdiagnose, das Tabakrauchen, Hauptbeschwerden, Verlauf der Erkrankung, das Vorliegen von Allergien, Obstruktion, bronchiale Hyperreagibilität oder das Ansprechen auf Kortikosteroide kombiniert mit der körperlichen Untersuchung, häufig gar nicht innerhalb der zeitlichen Vorgaben abzuarbeiten.

Auch die Initiierung und die Interpretation anschließend durchgeführter Diagnostik wie Lungenfunktionstest und gegebenenfalls Bildgebung benötigen Zeit, die häufig im Alltag nicht zur Verfügung steht.

Die «Übersichtsarbeit» dieses Heftes «Personalisierte Medizin bei Asthma und chronisch-obstruktiver Lungenerkrankung» von Liam Heaney und Lorcan McGarvey beschäftigt sich ausführlich mit dieser Problematik und stellt nochmals heraus, dass das Verstehen des klinischen Problems eine Grundvoraussetzung für eine personalisierte Patientenversorgung ist, und die genaue Diagnose, die sich eben häufig tatsächlich erst maßgeblich durch das Gespräch, die Fragen, also die Anamnese insgesamt erschließt. Die Anamnese beinhaltet ebenso die Eruierung von Komorbiditäten bei den obstruktiven Lungenerkrankungen.

Auch im Fallbericht in der Rubrik «Erfahrung aus der Praxis», der sich mit offenen Fragen zur endoskopischen Lungenvolumenreduktion (ELVR) mit Endobronchialventilen beschäftigt, geht es letztendlich im weiteren Sinne um die Präzisionsmedizin. Denn auch hier gilt es herauszuarbeiten, welche Patienten ganz gezielt von dieser Art der Therapie profitieren. Ein Einzelfall stellt hierbei die Diskussionsgrundlage dar und es gilt, auch hier die Patienten sehr genau auszuwählen, die einer endoskopischen Lungenvolumenreduktion zugeführt werden. Des Weiteren macht dieser Fall deutlich, dass eben auch konsequent über therapeutische Komplikationen aufgeklärt werden muss. Es ist mittlerweile gut belegt, dass die ELVR als semiinvasive Technik bei geeigneten Kandidaten mit fortgeschrittenem Lungenemphysem die Symptomatik verbessern kann. Die Belastungstoleranz und auch die Lungenfunktion werden bei entsprechender Patientenauswahl günstig beeinflusst. Es ist aber von herausragender Bedeutung auch Komplikationen wie Pneumothorax, Exazerbationen oder, wie im hier vorliegenden Fall beschrieben, das starke Abknicken des Bronchus anzusprechen.

\section{KARGER}

Fax +497614520714 information@karger.com www.karger.com (c) 2018 S. Karger GmbH, Freiburg

Accessible online at: www.karger.com/kkp
Prof. Dr. Andreas Rembert Koczulla

Professur für pneumologische Rehabilitation, Chefarzt Fachzentrum für Pneumologie, Schön Klinik Berchtesgadener Land

Klinik für Pneumologie, Philipps-Universität Marbur

Biegenstraße 10, 35037 Marburg, Deutschland

koczulla@med.uni-marburg.d 
Sicherlich ist die Präzisionsmedizin eine sinnvolle Beschreibung von Medizin, wie sie sein sollte, und wie sie in Zukunft sicherlich auch sein wird, die die Individualität des einzelnen Patienten herausstellt und die Definition der Individualität des einzelnen Patienten durch den Arzt - sprich: die genaue Anamnese, Untersuchung und Einordnung der weiteren Diagnostik - als immense Herausforderung verdeutlicht.

Bei aller technischer Entwicklung sei aber darauf hingewiesen, dass eben schon 30 Jahre alte Daten zeigen konnten, dass die Anamnese eine exzellente Basis zur Einleitung der Präzisionsmedizin ist, die sicherlich durch viele neue innovative diagnostische Verfahren

\section{Literatur}

1 Peterson MC, Holbrook JH, Von Hales D, et al.: West J Med. 1992;156(2): 163-165. ergänzt werden kann, um den Patienten schließlich einer hoch individualisierten, zeitgemäßen Therapie zuzuführen.

Ich wünsche Ihnen nun, liebe Leser, bei den in diesem Heft versammelten Artikeln eine interessante Lektüre.

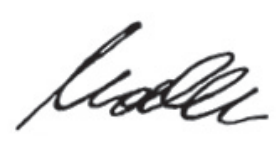

Prof. Dr. med. Andreas Rembert Koczulla 